\title{
Omics Studies and Systems Biology Perspective towards Abiotic Stress Response in Plants
}

\author{
Jyoti Prakash Sahoo1*, Laxmipreeya Behera1 ${ }^{*}$, Siddhartha Shankar Sharma², Jannila Praveena ${ }^{3}$, \\ Suman Kumari Nayak ${ }^{4}$, Kailash Chandra Samal1
}

${ }^{1}$ Department of Agricultural Biotechnology, Odisha University of Agriculture and Technology, Bhubaneswar, India

${ }^{2}$ Department of Genetics and Plant Breeding, Uttar Banga Krishi Viswavidyalaya, Cooch Behar, India

${ }^{3}$ Department of Fruit Science and Horticulture Technology, Odisha University of Agriculture and Technology, Bhubaneswar,

India

${ }^{4}$ Department of Plant Physiology, Odisha University of Agriculture and Technology, Bhubaneswar, India

Email: `jyotiprakashsahoo2010@gmail.com, ^klaxmipreeya1@gmail.com

How to cite this paper: Sahoo, J.P., Behera, L., Sharma, S.S., Praveena, J., Nayak, S.K. and Samal, K.C. (2020) Omics Studies and Systems Biology Perspective towards Abiotic Stress Response in Plants. American Journal of Plant Sciences, 11, 2172-2194.

https://doi.org/10.4236/ajps.2020.1112152

Received: November 7, 2020

Accepted: December 28, 2020

Published: December 31, 2020

Copyright $\odot 2020$ by author(s) and Scientific Research Publishing Inc. This work is licensed under the Creative Commons Attribution International License (CC BY 4.0).

http://creativecommons.org/licenses/by/4.0/

\begin{abstract}
Plant abiotic stress responses are vital yield-restricting aspect in agriculture. Recent technology in plant biology allows research of such stress responses at a molecular scale in plants. Network analysis provides in-depth knowledge regarding omics information visualisation as it reduces the intrinsic intricacy of such data. The use of integrated functional genomics helps to understand the relationship between the genomic profile and the phenotypic profile in different environmental conditions of an organism. Plants' responses to abiotic stress are often considered as a complex process. Systems biology approaches allow visualising and understanding how plant life works to overcome abiotic stress. The combination of integrated functional genomics along with bioinformatics will put a hand in additional in-depth research knowledge on stress tolerance to plants by exploiting available genetic information and continuously improving techniques and strategies. Most of the omics technologies are high throughput with very rapid data generation rates and humongous outputs. These technologies have made noticeable contributions to the modern-day improvements in our knowledge of plant biology. So, in this review, omics studies and the system biology approach towards abiotic stress tolerance in plants are highlighted.
\end{abstract}

\section{Keywords}

Genomics, Proteomics, Transcriptomics, System Biology, Abiotic Stress, Plants 


\section{Introduction}

A unit of self-sustaining but interconnected components that function together as a unified whole is referred to as a system [1]. In order to sustain the functional stability of a system, the member components of that system need to be functional together coherently. Such systems can be identified at different stratum in the biological world such as biological ecosystems, organisms, organ system, tissue system, cellular system, genes, proteins and metabolites. The borderline of these systems is often vaguely described owing to their connections at a multi-scale level. Thus studying the interaction at the level of a single system becomes more and more complex, further complicating the studies at multiple strata or hierarchical levels. The last decade has witnessed an increase in the knowledge and research in the systems biology science, which integrates the humongous data generated from the genomic technologies, projects computational models thereby helping us to figure out the topology, dynamical functions of the molecular systems sustaining an organism [2]. Once a model is projected this field of systems biology research then proceeds with data-driven as well as hypothesis-driven forward approaches (Figure 1). The advantage here is it clubs based descriptive approaches based on data integration predictive approaches based computational simulation that helps biologists to retrieve information from data gathered with the multitude of omics technologies and synthesised network models and also to study their response and pattern of growth in a simple and predictive manner. This approach brings down differences between biological studies and integrates them with computer science, mathematics, physics and chemistry. However, the essence of this area of study is still under debate over it to be a multidimensional data analysis field or dynamic modelling technique [3].

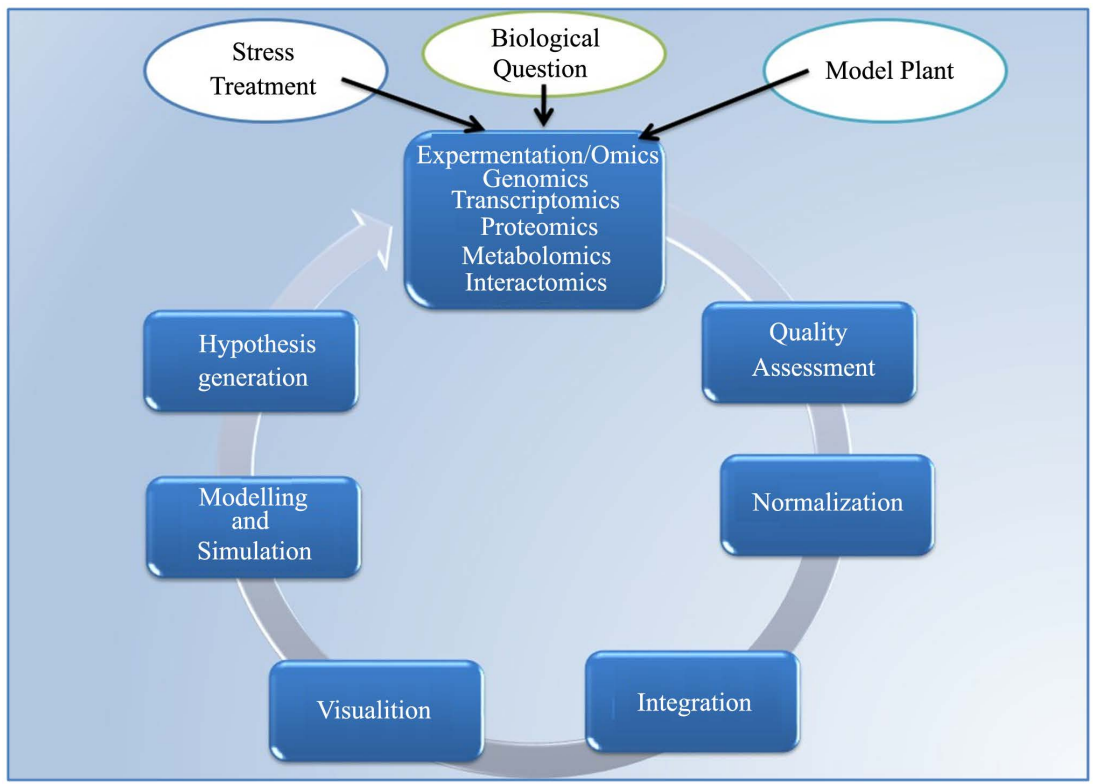

Figure 1. A general model of a biological system approach for plant stress response. 
Plants are devoid of escaping as they are sessile and thus face the environmental condition as it is. Due to this, evolution has helped them to develop flexibility in their reaction to environmental stress caused by both abiotic as well as biotic factors like pathogen and pest attacks, sunlight/UV, drought, temperature, and nutritional stress. This flexibility refers to the genetic potential of the plant optimised for its survival in a wide range of environmental stress. Duplication events at the genome level in the past have led to the diversification of duplicated genes and pathways. Even such events and their analysis are not simple as they are complicated and the variable environment will elicit complex responses resulting from a different group of pathways and their components. This is also affected by the growth stage and the nutritional status of the system involved. Because of such complexities studying responses at the molecular level with the help of transcript and protein profiling is a difficult task to follow. Biological research has always been an integrative approach with the involvement of physiological, morphological, molecular, biochemical and genetic information pieces as seen in the case of plant breeding and ecological studies [4]. System biology studies have extended this practice to genome-scale, offering answers to complex issues by enabling virtual test and analysis and hypothesis testing [5]. The research at present is focused on to model stress response mechanisms of plants and to define their effect on many such different plant processes [6]. Recently these efforts are reinforced by an extensive range of software [7], made to develop an advanced and intuitive projection of the experimental data, and for designing networks and models that can allow users to generate new hypotheses for future research. The principal needs for plant growth are carbon source, energy (light), water, and mineral nutrient. Abiotic stress is defined as physical environmental conditions that hamper the normal plant life processes, thereby reducing growth as well as yield below optimum levels. And the response of a plant to such stresses can be dynamic and complex [8] [9]; as they can be both elastic (reversible) and plastic (irreversible). The effect of stress response faced by a plant depends on either the plant tissue or an organ as a whole. For example, transcriptional responses to stress in roots are specific, distinctive and are quite diverse depending on the stress involved [10]. The level and duration of stress (acute vs chronic) also have a significant effect on the nature of the response [11] [12]. Water stress or water deficit can inhibit plant growth by decreasing water uptake by the expanding cells, as a result altering the enzymatic and rheological properties of the plant cell wall; for instance, by the activity of ROS (reactive oxygen species) on the enzyme of the cell wall. Rather than this, water stress also affects the interaction of cell wall components; calcium and pectate and ultimately reacts on cell wall non-enzymatically [13]. Furthermore, the activity of aquaporin and xylem embolism also affect the water conductance to the expanding cells [14]. The initial water stress primarily inhibits growth before hampering the process of photosynthesis or respiration [15]. To study all these mechanisms, it is essential to know the process of abiotic stress response in plants. So, in this review, the different omics approach towards abiotic stress re- 
sponse in plants is elaborated along with the integrated system biological approaches which will help to understand the perspective towards abiotic stress response in plants.

\section{Genomic Study of Plants in Response to Abiotic Stress}

The changing environmental conditions lead to trigger an extensive range of abiotic stress responses in plants, which may affect growth inhibition due to changing of transport and metabolic processes. Among other stresses, ion imbalance and hyperosmotic stress primarily influence by abiotic stress. Abiotic stress can increase the production and accumulation ROS, which was not at all good and may be detrimental to plants at higher concentrations and ultimately results in oxidative stress. This oxidative stress brings a significant imbalance in a cell compartment and leads to serious physiological challenges. Excess production of ROS causes damage to the biomolecules like proteins, carbohydrates, lipids, DNA and sometimes leading to apoptosis (Figure 2). Rather than this commencement of stress can also trigger some initial sensors (mostly unknown) which result into activation of the cytoplasmic Calcium ions and protein signalling pathways that show the way to physiological changes through stress-responsive gene expression. In case of both in vivo (greenhouse, field) and in vitro (tissue cultures) situation plant expose to different environmental stress such as water stress, osmotic and salinity stress, temperatures stress, wounding, light conditions, and atmospheric variables is of utmost importance. The plant response is unusual and complexed as it triggers and influences the integration of stress effects and responses at diverse underlying levels of organisation over space and time [16]. Under field conditions, these responses are often synergistically or antagonistically affected by the superimposition of additional stresses. Mainly, drought and salinity are two major abiotic factors that draw up the boundaries of crop productivity [17].

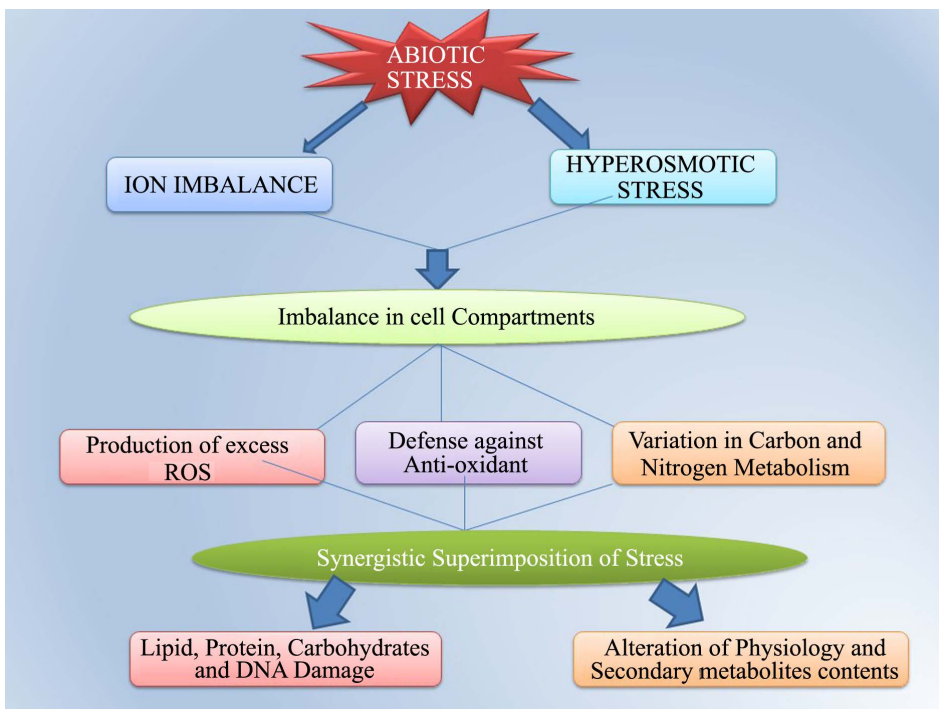

Figure 2. Physiological and Biochemical Basis of abiotic stress on plants. 
To enhance crop productivity, it is important to recognise the mechanism of plant responses to environmental stress (Figure 3). All those abiotic stress in no way works alone and a complicated mechanism of diverse interacting environmental elements contributes at varying ranges to the general stress. The transduction pathways for osmotic and different environmental stress responses are probably to be very complex and could contain signal molecules which include ABA, cyclic nucleotides, and inositol polyphosphates. Thus, the best mechanism(s) by which plant life responds to drought or excessive salinity stays unresolved. Consequently, engineering genes that defend and preserve the character and shape of cell additives can enhance tolerance to stress. However, on the molecular level, the maximum of the modifications are probably the result of changes with inside the expression of genes. Therefore, it is critical to discover the applicable genes and represent their regulation in reaction to water and/ or salinity stress [18].

\section{1) Gene expression in response to drought stress}

When plant exposed to drought, a large number of genes regulation were identified. To get the in-depth knowledge about the specific function of these genes and their role in plant adaptation to water scarcity a broad range of tools, from gene expression patterns to the use of transgenic plants are being utilised. Many physiological parameters that structure out the plant adaptation to drought stress such as root size and depth, phenology, hydraulic conductivity and the storage of reserves are usually linked with structure and development of plant are more constitutive rather than stress-induced.

Rather than this tolerance to drought in plant largely lies in its ability to get rid of surplus radiation, concomitant stress under natural conditions. The physiological and molecular changes responsible for leaf photo-protection, mainly

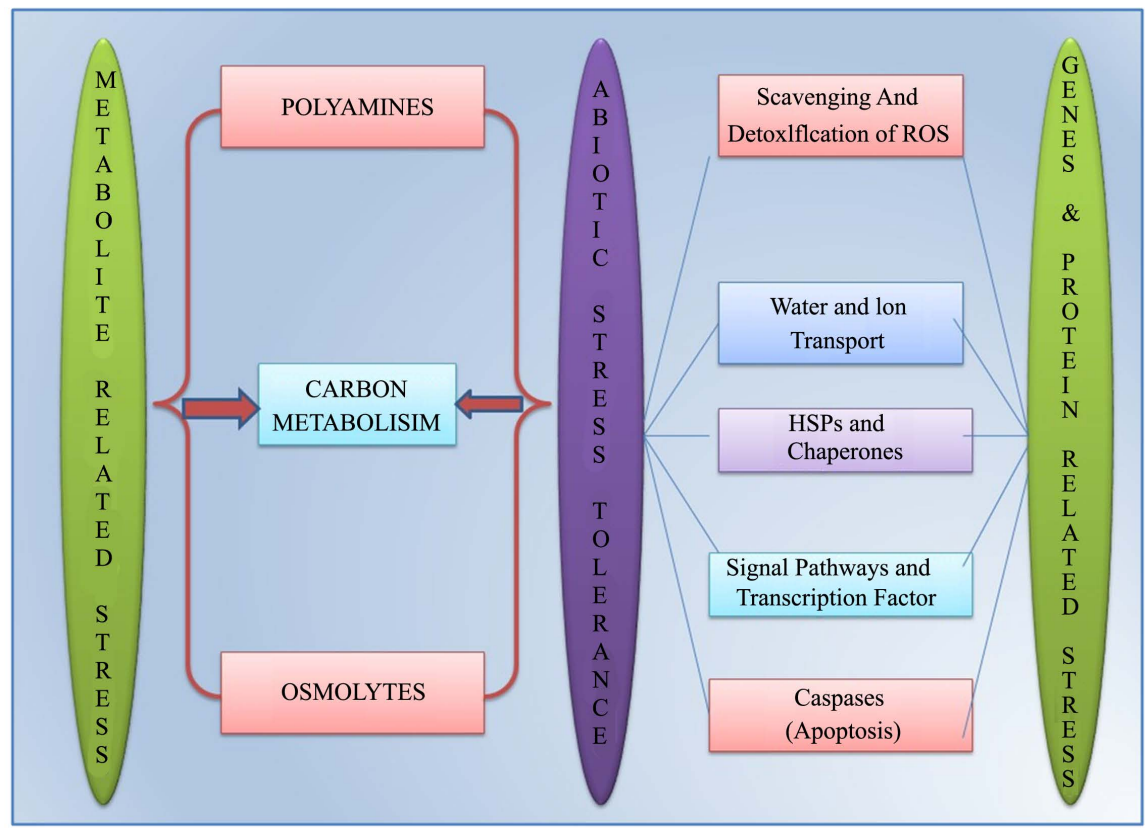

Figure 3. Molecular basis of abiotic stress in plants. 
those in relation to thermal dissipation, and oxidative stress is being actively researched. The results revealed that most of the drought-regulated genes are also responsive to light (KNAT3, KNAT4, SEN1, DIN9, DIN10, and ACP4) and/ or circadian rhythm (e.g., CCA1, WNK1, and FSD1), indicating towards the fact that drought might affect a plant's light and circadian cycles and/or vice versa [19]. Rather than this, some drought-regulated genes are also responsible for multiple stresses and stimuli. For example, RD29B, COR47, and ERD14 have been identified to be both responsive to water deficiency and low-temperature stress; while RD20 and RD22 are identified as responsive to salinity and desiccation; VSP2 is suggested to be responsive to high temperature, oxidative stress, insect, aridness, salinity and wounding. It was reported that 67 genes that shown responses to all nine stress treatments (cold, osmotic stress, salt, drought, genotoxic stress, ultraviolet light, oxidative stress, wounding, and high temperature) [20] while RHL41 (At5g59820) only revealed responsive against salinity, wounding, heat, cold, oxidative stress, light stimulus and chitin. In recent times, emerging microarray technology has been used to discover stress-responsive genes in Arabidopsis [20]. To a certain extent, this technology has also been employed to study soybean gene transcription under water stress in root and shoot (leaf) tissues at vegetative stage. For improvement of the stress tolerance of plants by gene transfer, mostly stress-inducible genes were used [21]. Even though hundreds of genes are identified to be involved in abiotic stress response only a few numbers of them have been well characterised [22], the functions of the remaining genes unclear and there are probably still more genes yet to be discovered.

\section{2) Gene expression in response to salinity stress}

There are numerous evidences illustrating the changes in the gene expression occurring in plants in the aftermath of exposure to salt. During the last decade, a large number of salt-responsive genes have been identified and analysed. Salinity tolerance exists in a broad range, from very high plants (Beta vulgaris) to extremely low plants (Citrus spp.). Salt-tolerant genes were identified from salt tolerance species gives a direction to a better understanding of the mechanisms that differentiate them from their salt-sensitive counterparts. A number of different approaches have been undertaken to identify genes whose expression is influenced by salinity in plants. A plethora of techniques has been employed to identify and isolate genes whose expressions are influenced by salinity. Most of these experimental designs involve screening cDNA libraries that have been isolated from plants cells exposed to salt treatment. Differential screening with probes that are derived from mRNA isolated from salt-stressed and non-stressed plant tissue is one of the most successful methods used to isolate salt-responsive cDNA clones from these libraries. Nowadays, salt-sensitive mutants of yeast used cDNAs encoding proteins increase the salt tolerance.

According to Pulla and coworkers [23] reported that cDNA clone having an S-adenosyl-L-methionine synthetase (SAMS) gene, called PgSAM (isolated from a commercial medicinal plant called Panax ginseng), gives the protection against 
different abiotic stresses. The prediction towards the encoding of a precursor protein of 307 amino acid residues may share high homology with a number of other plants SAMS [24]. There was a significant homology between the partial amino acid sequence of the protein and the nucleotide sequence of CSA with the mammalian glutathione peroxidase (GP). There was an increased level of expression of Cit-SAP in NaCl-treated cultured citrus cells as well as in citrus plants irrigated with saline water, its homology to GP point out to the possibility that salt stress may be increasing the production of free radicals. Another study in rice (Oryza sativa) using cDNA microarray including about 1700 independent cDNAs was carried out to recognise cold, drought, high salinity, and/or ABA inducible genes. This analysis identified a total of 73 genes as stress-inducible candidate genes of which 62, 57, 43 and 32 genes were induced by drought, high salinity, $\mathrm{ABA}$ and cold respectively. Out of these 73 stress-inducible rice genes, 51 have already been reported in Arabidopsis with a similar function or a gene name. Transcriptome analysis also revealed novel stress-inducible genes, suggesting some differences between Arabidopsis and rice in terms of their response to stress [25].

\section{3) Gene expression in response to nutrient stress}

Amount of nutrient and mineral present interfere with the regular plant growth and development. Therefore one of the crucial goals of the plant breeding is to achieve high nutrient potency. Transcription analysis and micro array studies of 6172 genes in shoots and roots of Arabidopsis performed to check with macronutrients (like Nitrogen and Phoshporus), and/or microelements (like iron or Zinc) associated with P depletion. It was reported that near about $30 \%$ of these genes (nearly 1835 genes) were shown up- or downregulation by two folds or more during the first $72 \mathrm{~h}$ under Phosphorus deprivation. These genes show a broad range of functions. In roots system more number of genes show downregulation (296 genes were specifically inhibited and 141 specifically upregulated) where as in shoot system the reverse occurred (617 downregulated and 488 upregulated genes) [26]. It's significant to point out that an adaptative reprogramming undoubtedly desires the more of downregulated genes than upregulated genes. Expression and signal transduction pathways embody transcription factors, macromolecule kinases, and enzymes related to phosphoinositide metabolism [27]. For instance, a 104-kDa macromolecule, SAP 104 (that accumulates in rice seedlings in response to several abiotic stress conditions) and immunological homologues of rice SAP 104 have been detected in many species both monocots and dicots. It might be projected that plants experiencing stress would require a strong protein turnover machinery that can degrade stress-damaged and environmentally regulated proteins.

\section{4) Gene expression in response to heavy metal stress}

Despite the fact that much of the progress made in the analysis of gene expression done at the mRNA levels have shared a depth understanding of plant's response to heavy metals, there are still many avenues to be discovered regarding the functional translated portions of a plant genome. A number of cellular regu- 
latory proteins or signalling proteins participating in cell growth, apoptosis were targeted due to the carcinogenic effect of metals. Some factor was responsible for the expression of protective genes involved in DNA repair, powering immune system, restricting the proliferation of damaged cells, and inducing apoptosis [28]. Reported by many previous researchers most toxic stresses are due to heavy metal exposure that affects gene expression, through the modulation of genes such as NF-kB, AP-1, and p53 activities. Plants under heavy metal stress respond via distinct mitogen-activated protein kinase (MAPK) pathways, cellular signalling mechanisms in roots due to an increased level of copper and cadmium ions. For instance, it was seen in alfalfa (Medicago sativa) seedlings that the exposure to a higher amount of copper or cadmium ions activated four distinct MAPKs: SIMK, MMK2, MMK3, and SAMK were also reported [29]. Meta stress induces the formation of small metal-binding peptides called phytochelatins [30], they encode phytochelatin synthases and regulates the metal detoxification process in eukaryotes [31]. Phytochelatins also sequestered phytochelatin-metal complexes into the vacuoles to assist tolerance for heavy metal stress. Tonoplast-localized Abc2 transporter mediates phytochelatin by accumulation in vacuoles and thereby confers cadmium tolerance [32]. Expression of the serine acetyltransferase (SAT) gene family in Arabidopsis thaliana was studied in response to exposure to heavy metal cadmium (Cd). Expression of all the SAT genes was upregulated at different levels by $\mathrm{Cd}$ treatment in the leaf lamella, trichomes, root and stem cortex [33].

\section{Transcriptomics: A Key to Understanding Abiotic Stress Responses in Plants}

Transcriptome profiling study contributes to a transparent understanding of plant responses to abiotic stresses. Recently, transcriptome analysis aided by next-generation sequencing (NGS), RNA-seq for sRNAs and their relevance in genomics research, have improved plant genomic resources [34]. Five thousand three hundred sixty-five variably expressed probe sets were identified. Alamo, a switch grass cultivar opened to heat stress, exploiting switch grass Affymetrix gene chips [35]. By analysing transcriptomes under heat stress, 16 common genes have been identified in four monocots; switch grass, rice, wheat and maise. As most of them were associated with protein refolding processes; they can be exploited as valuable biomarkers for identifying heat-sensitive plant genotypes. Wakasa and co-workers worked on RNA sequencing-mediated expression profiling in the transgenic rice plants, produced as a result of homologous recombination, in which endogenous genomic OSIRE1 (ER stress sensor/transducer) was replaced by missense alleles that were defective in ribonuclease activity [36]. These results give direction towards mining valuable information about the ER stress response and the discovery of new ER stress-related genes. A comparison of plant faces under laboratory conditions and in the field are exposed to several types of stresses that claim agonistic or antagonistic responses or at the same time a number of potentially unrelated responses to a specific stress condition. A 
similar result was reported by Rasmussen and coworkers by comparing transcriptome changes in ten different Arabidopsis thaliana ecotypes under diverse stresses and their combinations [37]. This study revealed that a majority of the transcriptome changes i.e., $61 \%$, in response to double stresses were not projected from the responses to the single stress treatments. In light of this research, researchers have outlined co-expression network modules responding to single as well as combined stresses. Studies on RNA-seq analysis of Chenopodium quinoa under four different water treatments (field capacity to drought) exhibited an overlap among drought stress tolerance and other similar abiotic stress mechanisms [38]. Kudapa and coworkers [39] employed several Sanger EST collections of chickpea, along with sequence data from two different Next Generation Sequencing platforms (Illumina and FLX/454) of chickpea, to produce an extensive transcriptome assembly (CaTA v2). Additionally, NIPGR (National Institute of Plant Genome Research, India) has developed the Chickpea Transcriptome Database (CTDB), which can provide comprehensive datasets and information about the chickpea transcriptome (http://www.nipgr.res.in/ctdb.html). Apart from NGS, another technique; subtractive cDNA suppression hybridisation (SSH) technology has proved to be very effective in transcriptomic studies in revealing stress responses [40]. Transcriptome sequencing of chrysanthemum plants under dehydration stress using the Illumina sequencing also cleared the understanding of the molecular mechanisms of dehydration stress responses [41]. Zhu and coworkers [42] employed a comparative microarray analysis approach to explore the transcriptome changes in cotton under five different abiotic stresses. Their study revealed the functional genes and also the stress-related pathways. This suggested an interaction of responsive genes or pathways towards multiple abiotic stresses in the cotton seedlings. Transcriptomic technologies can be exploited to provide a deep understanding and unbiased representation of the transcript datasets, which is very crucial in non-model plants that lack genome sequence information [43]. However, the frequent inconsistency between protein levels and loads of cognate gene transcripts suggests the requirement of complementary analysis of the proteome for future validation of candidate genes and their pathways [44].

\section{Proteomics: A Closer Look at Translatome Regulating Cellular Responses}

Basically, gen-Omics studies depend on transcriptome analysis over ever-changing external conditions so as to explore transcripts that are differentially regulated, and sometimes it is hypothesised that changes in transcript levels might result in corresponding changes in macromolecule levels. However, it has been shown by many workers that macromolecule levels don't usually essentially corresponds to messenger RNA levels [45] [46]. Anderson and co-workers [47] reported that there is a very low level of correlation in quantity between mRNA and protein abundance. Protein level can be regulated by changing either the rate of synthesis, stability of the m RNA, or the stability of the protein itself [48]. The study of 
translatome is in reference to the pool of all RNAs that are present with ribosomes purified using an affinity tag. Study of the translatome or proteome can confirm the presence as well as gives a direct measure of the quantity present. Proteins are the functional entities or translated portion of the genome that play a crucial role in plant stress response. It allows us to understand the protein networks and metabolic pathways involved in stress tolerance mechanism.

Identification of a master regulator protein that plays a key role in the abiotic stress response pathway is of utmost importance for developing or modelling a genetically engineered crop so as to allow us to understand stress response. Halbeisen co-workers [49] analysed the transcriptome along with the translatome in yeast cells which were exposed to different stresses, to study the discrepancy between transcripts and the protein levels. Their analysis suggested that transcriptome and translatome show a strong coordinated response, particularly under severe stress. In $A$. thaliana, an investigation was undertaken to study the translational regulation under control and sublethal hypoxia stress conditions by precise mapping of ribosome footprints (RFs) on mRNAs, the results showed that there was nearly 100 -fold variation in the efficiency of translation of the mRNAs under each condition. Such studies have illuminated scientific research about the peculiarity of posttranscriptional and translational regulation controlled by a lower level of external stress [50].

Translational efficiency of individual mRNAs of $A$. thaliana seedling after exposure to temperature stress was studied by Yanguez co-workers [51] using genome-wide analysis and they concluded that translation exerts a wide regulation on gene expression. Unlike some mRNAs where the translation is severely repressed, translation of homeostasis and also stress-related mRNAs do follow a differential pattern. Their work established that mRNAs with specific characteristic features, such low 5-UTR G + C content and small cDNA length, are translated preferentially. Proteomics have been extensively employed by researchers to solve the problem of heavy metal stress. Brassica juncea L. roots, under Cd stress, overexpress sulfite reductase and O-acetyl serine sulfhydrylase proteins because of the reduction of sulfate to its cysteine form [52]. Proteomics study involving leaf mesophyll tonoplast of Hordeum vulgare L, revealed an MRP-like ABC transporter along with two novel CAX transporters (CAX1a and CAX5), indicating $\mathrm{Cd}^{2+}$ transport into the vacuole [53]. Additionally, in Glycine max L. leaf samples, the abundance of both Hsp70 as well as peroxiredoxin were reported [54] and up-regulation of $\mathrm{Cd}^{-}$chelating pathways proteins were reported in different plant species viz. A. thaliana, G. max and Linumus itatissimum, . [55] [56] [57] [58]. Similar work has also been carried out in Lupinus albus roots with B deficiency, where proteins that are involved in cell division or metabolic processes were down-regulated [59]. Increased expression of proteins involved in ROS detoxification, defence responses, photosynthesis and chloroplast organisation were reported in Zea mays under Cr stress [60]. 


\section{Metabolomics: Pinning Down the Stress-Responsive Pathways}

In recent years, metabolOmics provided a new horizon to plant stress-related studies and has become an indispensable tool in deciphering the molecular mechanisms underlying stress responses [61]. Exposure to stresses makes the plant metabolism to undergo reconfiguration to maintain the metabolic homeostasis and generation of compounds that face the stress. In light of recent developments, like metabolOmics and systems biology approaches, detailed information about the crucial components of metabolic pathways have been obtained. Metabolomic studies of $A$. thaliana plants studied under drought stress has revealed that the accumulation of different metabolites, such as amino acids such as gamma amino butyrate (GABA), proline, tricarboxylic acid (TCA) cycle metabolites and raffinose family oligosaccharides, and which are known to be produced in response to drought stress in plants [62]. ABA-dependent transcriptional regulation which is responsible for activation of many stress-related metabolic pathways, have been reported using mutant and transcriptomic approach [62]. Metabolite profiling has revealed a temporal distinction in $A$. thaliana leaves when subjected to mild osmotic stress [63]. Correlating with the transcriptional response matured leaves expressed typical drought-responsive genes like proline, erythritol and putrecine that gradually decreased in expanding leaves [64]. The same was observed in metabolic studies that pointed out the variable response and degrees of desiccation of plant metabolism temporally. As expected, amino acid metabolism showed that most of the amino acids accumulated in severely desiccated leaves as compared to mildly desiccated leaves.

Metabolite profiling done in maise and wheat when exposed to water stress conditions suggested a common change in the levels of metabolites like branched-chain amino acids (BCAAs) [65]. Verslues and Juenger [66] reported an important role of metabolic regulation that pointed out the regulation of photosynthesis and accumulation of osmolytes as a drought stress response in $A$. thaliana. Caldana and co-workers [67] analysed transcriptome and metabolome profiling of $A$. thaliana subjecting it to eight different environmental conditions. Metabolic response under high light displayed an accumulation of photorespiratory intermediates like glycine and glycolate in the early phase. Interestingly, they have also reported similar responses during the mid-phase of high light stress and low-temperature treatments, including accumulation of fructose, phenylalanine and shikimate, and a decrease in succinate. However, the physiological basis of such overlapped responses is currently not well understood. Kusano and co-workers [68] analysed the effect of UV light on $A$. thaliana metabolism and reported a biphasic response. In the early phase of stress, major changes in levels of primary metabolites, including ascorbate derivatives, were seen. Classically defined UV-B protectants, such as flavonoids and phenolics, showed mid- to late-term responses. Cell priming upon early exposure to UV-B involving reprogramming of the metabolism can be suggested for efficient diver- 
sion of carbon towards aromatic amino acid precursors of the phenyl propanoid pathway [69].

Metabolite profiling done in $A$. thaliana leaves has helped in explaining the metabolic basis of darkness induced senescence and the function of mitochondrial alternative; electron transport pathway under dark treatment [70]. In other metabolite profiling studies, an increase in BCAAs, i.e. leucine, valine, isoleucine, and other amino acids sharing synthetic pathways with BCAAs i.e. threonine, lysine and methionine were reported under abiotic stress conditions [71]. The authors have suggested BCAAs function to be as compatible osmolytes since drought stress leads to an increase in accumulation of BCAAs in the plant tissues. Interestingly, protein degradation also serves as an alternative respiratory substrate for plants under stress [72]. All of these techniques have greatly increased our understanding related to candidate genes, proteins and their pathways playing major roles in plant stress responses but there is still a long arena to be explored. More recently, researchers have tried to combine either two or all three Omics approaches to have a holistic view of stress responses [73]. Zeng and co-workers [74] have combined these Omics approaches to study the alkaloid biosynthesis in Macleaya sp. Phosphate-deficient studies in A. thaliana roots done by Lan co-workers [75] showed multiple levels of gene regulation thereby suggesting integrated measurement, interpretation of changes in protein and transcript is sufficient for generating a complete package of the components critical for stress responses.

A data warehouse of maise; OPTIMAS-DW has been developed by Colmsee and co-workers [76]. It is capable of handling various data domains, integrates data from these domains (such as metabolomics, ionomics, transcriptomics, proteomics, phenomics) and enables the user to explore different systems biology questions. Amiour and coworkers [77] studied the potential use of 'Omics' studies for a better understanding of whole-plant nitrogen. Srivastava and co-workers [78] put forward a data evaluation strategy to derive an efficient way of compiling complex and multi-platform datasets to obtain major biological information in transgenic Populus plants harbouring superoxide dismutase gene. It provided system-level information on the responses to oxidative stress and ROS metabolism.

\section{Systems Biology Approach towards Abiotic Stress Response in Plants}

\section{1) Co-expression analyses identifying regulatory hubs}

Investigation of co-expression of candidate genes utilising online, analytical scientific instruments, like ATTED-II [79], is a significant utilisation of transcriptomics data. For knowing consistency ties and finding major genes, this methodology is extremely stimulating. MYB transcription factors managing glucosinolate biosynthesis in Arabidopsis were identified by Hirai and coworkers [80] owing to $\mathrm{S}$ and $\mathrm{N}$ deficiency approach of metabolomics and transcriptomics. In glucosinolate digestion, genes and also the metabolites were discov- 
ered to be coordinately controlled [81]. A quality co-articulation network investigation of 1094 microarrays of Arabidopsis was conducted by Mao and coworkers using a non-target methodology and recognised 382 modules [82].

Photosynthesis, protein unity and response to oxidative stress were three primary components with the most hubs. Using the InferGene software programme, Carrera and coworkers [83] analysed the Arabidopsis genome to develop a regulatory model for it. Ten numbers of genes, including $K A N 3$ (auxin), MYB29 (gibberellin), MYB121 (abscisic acid), ERF1 (ethylene) and ANAC0366 (stress response TFs), were projected as the most relevant expressed genes in the Arabidopsis genome. While researching the drought response, Lorenz and coworkers [84] found a number of hubs in transcriptional units of loblolly pine roots. An inositol transporter gene thioredoxin, a cardiolipin synthase or phosphatidyl transferase gene, 9-cis-expoxycarotenoid dioxygenase gene, SnRK2 kinase gene and zeatin O-glucosyltransferase gene have been the highly marked and ranked hubs established [84]. The key regulators of the pathway of phospholipid metabolism, the signalling pathway of ABA biosynthesis and the pathway of cytokine metabolism are all these genes. The co-expression analysis was examined by Weston and coworkers [85] to find a way to identify six abiotic stress reaction modules in Arabidopsis, including Calcium signal transduction genes and ankyrin-repeat protein genes. Using this approach and analysis, tests were conducted to investigate the response of heat and light on various plant species and evidence were reported involving heat shock proteins, heat tolerance, ROS, photosynthesis and metabolism of oligosaccharides [86].

2) Revealing multiple phases in stress responses through time-series analyses

The time series analysis enables one to differentiate between primary and secondary stress response. In such a transcriptomics study of time series of 7 abiotic stresses on different Arabidopsis strains [87], a crucial set of genes were articulated for non-specific reactions to every stress which included the transcription factors $Z A T 1, A Z F 2$ and $Z A T 12$. In the stress response, the readjustment of homeostasis is believed to be associated with this immediate response. Sun and coworkers [88] added a complexity metric of 9 various abiotic stresses to a set of Arabidopsis time-series information. They found that there were no longer 5 intergenic loci present and a greater density of cis-regulatory motifs in genes with a greater complexed metric. Most of the defined cis-regulatory motifs were linked to stress responses that were already known [88]. The consequences of hydrogen peroxide $\left(\mathrm{H}_{2} \mathrm{O}_{2}\right)$ signalling throughout the period, high light stress were reported by Vanderauwera and coworkers [89] using microarray experiments. They found that $\mathrm{H}_{2} \mathrm{O}_{2}$ was significantly involved not only in high light stress signalling but also involved in salinity stress, water shortages, extreme temperature. $\mathrm{H}_{2} \mathrm{O}_{2}$ has become a key regulator of the anthocyanin metabolic pathways with $70 \mathrm{kDa}$ heat shock proteins and many other genes. In plants, anthocyanins have an important role as an antioxidant. Highly regulated by $\mathrm{H}_{2} \mathrm{O}_{2}$ was a specific UDP-glycosyltransferase (UGT74E2). UGT74E2 responded very 
fastly to $\mathrm{H}_{2} \mathrm{O}_{2}$ and glycosylated indole-3-butyric acid (IBA) in a subsequent study [90], modifying plant morphology, auxin homeostasis and improving salinity and water shortages stress tolerance. In addition, auxin was also found to interact with the $\mathrm{ABA}$ thus raising the plant's $\mathrm{ABA}$ sensitivity.

Polymerase silencing (ADP-ribose) improved the greater threshold to light stress in Arabidopsis [91]. Reduced oxidative stress and enhanced energy use efficiency were part of the increased abiotic stress tolerance [92]. Kusano and coworkers [93] conducted a time-series experiment utlising both metabolomics as well as transcriptomics analyses on the effects of Ultraviolet-B light on Arabidopsis. They found that plants responded in two stages by upregulating primary metabolites during the first stage and by producing secondary defensive metabolites, in phenolics, in the second phase. Phenolic induction corresponded to transcripts that were participating in the phenylpropanoid pathway. However, the primary metabolism transcripts were less stable, showing that some mechanisms could regulate this pathway [94].

\section{Integration of Omic Approach}

The integration of transcript analysis and metabolite profiling has accomplished some convergence of methods; it could be most easily documented in this way to represent the reasoning of those downstream effects of changed transcription [95]. The increasing focus on metabolomics research is evident in the generation of metabolite detectors and repositories that integrate metabolite profiling and transcript profiling [96]. The lack of information on metabolomic flux parameters [97], that could be mitigated by the use of new powerful tools and procedures for imaging of NMR, which is a known weakness at present [97]. Experiments should have based on the performance of protein molecules or groups of proteins [98]. Specific profiling frameworks can bring together information on processes dependent on abiotic stress that must be fit into a plant-wide stress repository by bioinformatics to create novel phenotypes and also allows the exporting of this information to crop species. The genetic reaction of plants to abiotic stresses is usually described as a dynamic process based on the regulation of stress-related gene transcription (Figure 4). Recent results have, however, pointed towards new layers of complexity and regulation. In the plants exposed to abiotic stress, upstream biochemical molecules are responsible for the regulation of schedules and quantities of particular stress responses. The actual transcriptome that promotes the stress response is defined through post-transcriptional mechanisms compared to RNA processing, alternative splicing, as well as RNA silencing [98]. Many posttranslational adjustments like sumoylation and ubiquitination control the triggering of previously molecules existing outside regulatory proteins to maintain a rapid stress response. Besides that, there has been a bridge between these mechanisms that clearly demonstrates additional and overlaid levels of complexity in response to the environmental conditions. The system of posttranscriptional and posttranslational changes maintains downstream stress-related gene expression that are temporally and spatially appropriate. 


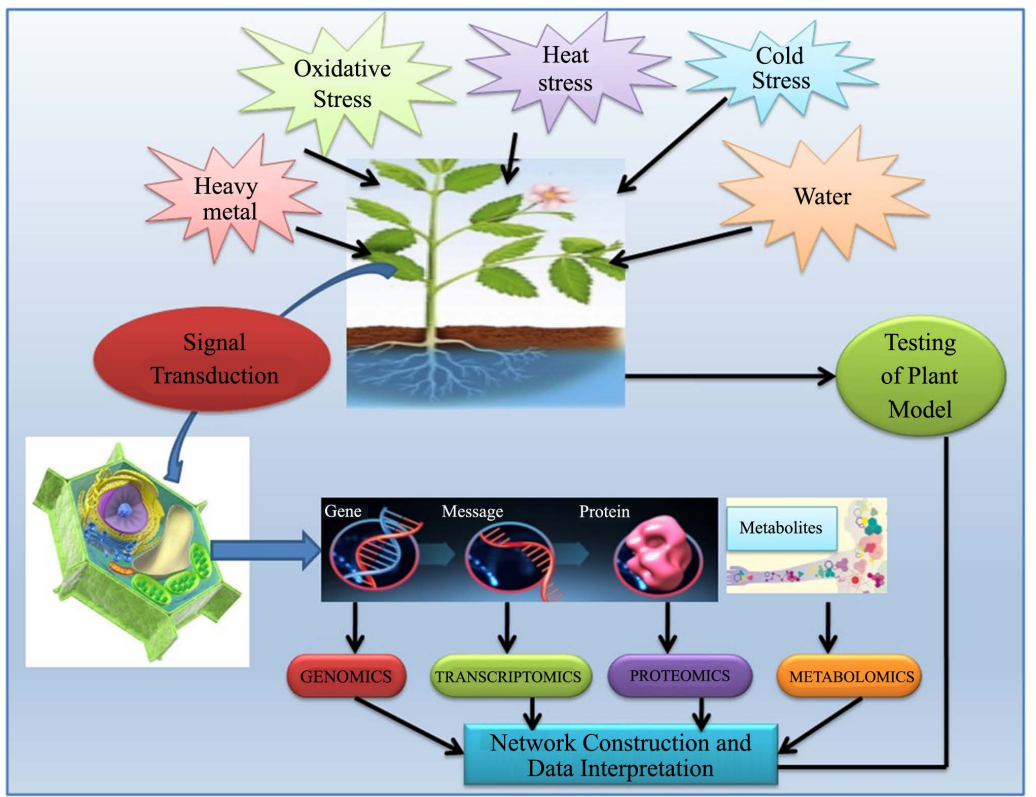

Figure 4. Integrated omics approach for abiotic stress management in plants.

Future crop engineering efforts may exploit insights from a better understanding of these new stress response regulation sites in order to generate abiotic stress-resistant plants.

\section{Conclusion}

In this review, the genomic studies, transcriptomic studies, proteomics studies as well as the metabolomics studies of plants in response to abiotic stress were discussed. Systems biology of plant stress responses calls for a massive quantity of genome statistics for the microarray research from samples mainly in diverse stress conditions. Methods like nuclear magnetic resonance and mass spectroscopy need to be advanced in addition to identifying a larger quantity of proteins as well as metabolites in plant samples. Computational strategies to make strong models that capture the complexities of plant biological processes also are required. In this review, the systems biology approach along with the integration of omic approach towards abiotic stress response in plants was also discussed. The aggregate of data integration and modelling can also additionally assist in making the "digital plant" grow to be a reality. The capacity to perform in silico experiments in such a plant will revolutionize the study of plant systems biology. The linkage of key regulatory locations or genes to its phenotypic tendencies will permit for fast development in genetic manipulation in crop plants. Omics research has accrued many statistics at transcript, protein and metabolite levels to understand the survival potential of plants in abiotic stress.

\section{Conflicts of Interest}

The authors declare no conflicts of interest regarding the publication of this paper. 


\section{References}

[1] Trewavas, A.A. (2006) Brief History of Systems Biology: "Every Object That Biology Studies Is a System of Systems.” Francois Jacob (1974). Plant Cell, 18, 2420-2430. https://doi.org/10.1105/tpc.106.042267

[2] Hammer, G.L., et al. (2004) On Systems Thinking, Systems Biology, and the in Silico Plant. Plant Physiology, 134, 909-911. https://doi.org/10.1104/pp.103.034827

[3] Kirschner, M.W. (2005) The Meaning of Systems Biology. Cell, 121, 503-504. https://doi.org/10.1016/j.cell.2005.05.005

[4] Sumner, L.W., Mendes, P. and Dixon, R.A. (2003) Plant Metabolomics: Large-Scale Phytochemistry in the Functional Genomics Era. ChemInform, 34, 817-836. https://doi.org/10.1002/chin.200319275

[5] Gutierrez, R.A., Shasha, D.E. and Coruzzi, G.M. (2005) System's Biology for the Virtual Plant. Plant Physiology, 138, 550-554. https://doi.org/10.1104/pp.104.900150

[6] Minorsky, P.V. (2003) Achieving the in Silico Plant. Systems Biology and the Future of Plant Biological Research. Plant Physiology, 132, 404-409. https://doi.org/10.1104/pp.900076

[7] Gehlenborg, N., et al. (2010) Visualisation of Omics Data for Systems Biology. Nature Methods, 7, S56-S68. https://doi.org/10.1038/nmeth.1436

[8] Skirycz, A. and Inze, D. (2010) More from Less: Plant Growth under Limited Water. Current Opinion in Biotechnology, 21, 197-203. https://doi.org/10.1016/j.copbio.2010.03.002

[9] Cramer, G.R. (2010) Abiotic Stress \& Plant Responses from the Whole Vine to the Genes. Australian Journal of Grape and Wine Research, 16, 86-93. https://doi.org/10.1111/j.1755-0238.2009.00058.x

[10] Dinneny, J.R., Long, T.A., Wang, J.Y., Jung, J.W., Mace, D., Pointer, S., Barron, C., Brady, S.M., Schiefelbein, J. and Benfey, P.N. (2008) Cell Identity Mediates the Response of Arabidopsis Roots to Abiotic Stress. Science, 320, 942-945. https://doi.org/10.1126/science.1153795

[11] Tattersall, E.A., Grimplet, J., Deluc, L., Wheatley, M.D., Vincent, D., Osborne, C., Ergul, A., Lomen, E., Blank, R.R., Schlauch, K.A., Cushman, J.C. and Cramer, G.R. (2007) Transcript Abundance Profiles Reveal Larger and More Complex Responses of Grapevine to Chilling Compared to Osmotic and Salinity Stress. Functional \& Integrative Genomics, 7, 317-333. https://doi.org/10.1007/s10142-007-0051-x

[12] Pinheiro, C. and Chaves, M.M. (2011) Photosynthesis and Drought: Can We Make Metabolic Connections from Available Data? Journal of Experimental Botany, 62, 869-882. https://doi.org/10.1093/jxb/erq340

[13] Boyer, J.S. (2009) Evans Review: Cell Wall Biosynthesis and the Molecular Mechanism of Plant Enlargement. Functional Plant Biology, 36, 383-394. https://doi.org/10.1071/FP09048

[14] Parent, B., Hachez, C., Redondo, E., Simonneau, T., Chaumont, F. and Tardieu, F. (2009) Drought and Abscisic Acid Effects on Aquaporin Content Translate into Changes in Hydraulic Conductivity and Leaf Growth Rate: A Trans-Scale Approach. Plant Physiology, 149, 2000-2012. https://doi.org/10.1104/pp.108.130682

[15] Hummel, I., Pantin, F., Sulpice, R., Piques, M., Rolland, G., Dauzat, M., Christophe, A., Pervent, M., Bouteille, M., Stitt, M., Gibon, Y. and Muller, B. (2010) Arabidopsis Plants Acclimate to Water Deficit at Low Cost through Changes of Carbon Usage: An Integrated Perspective Using Growth, Metabolite, Enzyme, and Gene Expres- 
sion Analysis. Plant Physiology, 154, 357-372.

https://doi.org/10.1104/pp.110.157008

[16] Blum, A. (1996) Crop Responses of Drought and the Interpretation of Adaptation. Plant Growth Regulation, 20, 135-148. https://doi.org/10.1007/BF00024010

[17] Misra, A.N., Biswal, A.K. and Misra, M. (2002) Physiological, Biochemical and Molecular Aspects of Water Stress in Plants, and Their Biotechnological Applications. Proceedings of the National Academy of Sciences of the United States of America, 72, 115-134.

[18] Liu, X. and Baird, W.V. (2003) Differential Expression of Genes Regulated in Response to Drought or Salinity Stress in Sunflower. Crop Science, 43, 678-687.

[19] Huang, D., Wu, W., Abrams, S.R. and Cutler, A.J. (2008) The Relationship of Drought-Related Gene Expression in Arabidopsis thaliana to Hormonal and Environmental Factors. Journal of Experimental Botany, 59, 2991-3007. https://doi.org/10.1093/jxb/ern155

[20] Swindell, W.R. (2006) The Association among Gene Expression Responses to Nine Abiotic Stress Treatments in Arabidopsis thaliana. Genetics, 174, 1811-1824.

https://doi.org/10.1534/genetics.106.061374

[21] Umezawa, T., Fujita, M., Fujita, Y., Yamaguchi-Shinozaki, K. and Shinozaki, K. (2006) Engineering Drought Tolerance in Plants: Discovering and Tailoring Genes to Unlock the Future. Current Opinion in Biotechnology, 17, 113-122. https://doi.org/10.1016/j.copbio.2006.02.002

[22] Zhu, J.K. (2002) Salt and Drought Stress Signal Transduction in Plants. Annual Review of Plant Biology, 53, 247-273.

https://doi.org/10.1146/annurev.arplant.53.091401.143329

[23] Pulla, R.K., Kim, Y.J., Parvin, S., Shim, J.S., Lee, J.H., Kim, Y.J., et al. (2009) Isolation of S-adenosyl-L-methionine Synthetase Gene from Panax Ginseng C.A. Meyer and Analysis of Its Response to Abiotic Stresses. Physiology and Molecular Biology of Plants, 15, 267-275. https://doi.org/10.1007/s12298-009-0030-x

[24] Holland, D., Ben-Hayyim, G., Faltin, Z., Camoin, L., Strosberg, A.D. and Eshdat, Y. (1993) Molecular Characterisation of Salt Stress-Associated Protein in Citrus: Protein and cDNA Sequence Homology to Mammalian Glutathione Peroxidases. Plant Molecular Biology, 21, 923-927. https://doi.org/10.1007/BF00027124

[25] Rabbani, M.A., Maruyama, K., Abe, H., Khan, M.A., Katsura, K., Ito, Y., et al. (2003) Monitoring Expression Profiles of Rice Genes under Cold, Drought, and High-Salinity Stresses and Abscisic Acid Application Using cDNA Microarray and RNA Gelblot Analyses. Plant Physiology, 133, 1755-1767. https://doi.org/10.1104/pp.103.025742

[26] Wu, P., Ma, L., Hou, X., Wang, M., Wu, Y., Liu, F., et al. (2003) Phosphate Starvation Triggers Distinct Alterations of Genome Expression in Arabidopsis Roots and Leaves. Plant Physiology, 132, 1260-1271. https://doi.org/10.1104/pp.103.021022

[27] Kaur, N. and Gupta, A.K. (2005) Sugar Signaling and Carbohydrate Metabolism under Abiotic Stresses in Plants. Current Science, 88, 1771-1780.

[28] Valko, M., Morris, H. and Cronin, M.T.D. (2005) Metals, Toxicity and Oxidative Stress. Current Medicinal Chemistry, 12, 1161-1208. https://doi.org/10.2174/0929867053764635

[29] Jonak, C., Kiegerl, S., Ligterink, W., Barker, P.J., Hukisson, N.S. and Hirt, H. (1996) Stress Signaling in Plants: A Mitogenactivated Protein Kinase Pathway Is Activated by Cold and Drought (Signal Transduction/Cold Stress/Salt Stress/Heat Stress). Proceedings of the National Academy of Sciences of the United States of America, 


\section{3, 11274-11279. https://doi.org/10.1073/pnas.93.20.11274}

[30] Ortiz, D.F., Kreppel, L., Speiser, D.M., Scheel, G., McDonald, G. and Ow, D.W. (1992) Heavy Metal Tolerance in the Fission Yeast Requires an ATP-Binding Cassette-Type Vacuolar Membrane Transporter. The EMBO Journal, 11, 3491-3499. https://doi.org/10.1002/j.1460-2075.1992.tb05431.x

[31] Clemens, E., Kim, E.J., Neumann, D. and Schroeder, J.I. (1999) Tolerance to Toxic Metals by a Gene Family of Phytochelatin Synthases from Plants and Yeast. The EMBO Journal, 18, 3325-3333. https://doi.org/10.1093/emboj/18.12.3325

[32] Mendoza-Cozatl, D.G., Zhai, Z., Jobe, T.O., Akmakjian, G.Z., Song, W.Y., Limbo, O., et al. (2010) Tonoplast-Localized Abc2 Transporter Mediates Phytochelatin Accumulation in Vacuoles and Confers Cadmium Tolerance. Journal of Biological Chemistry, 285, 40416-40426. https://doi.org/10.1074/jbc.M110.155408

[33] Howarth, J.R., Dominguez-Solis, J.R., Gutierrez,-Alcala, G., Wray, J.L., Romero, L.C. and Gotor, C. (2003) The Serine Acetyltransferase Gene Family in Arabidopsis thaliana and the Regulation of Its Expression by Cadmium. Plant Molecular Biology, 51, 589-598. https://doi.org/10.1023/A:1022349623951

[34] Varshney, R.K., Nayak, S.N., May, G.D., et al. (2009) Next-Generation Sequencing Technologies and Their Implications for Crop Genetics and Breeding. Trends in Biotechnology, 27, 522-530. https://doi.org/10.1016/j.tibtech.2009.05.006

[35] Li, Y.F., Wang, Y., Tang, Y., et al. (2013) Transcriptome Analysis of Heat Stress Response in Switchgrass (Panicum virgatum L.). BMC Plant Biology, 13, 153. https://doi.org/10.1186/1471-2229-13-153

[36] Wakasa, Y., Oono, Y., Yazawa, T., et al. (2014) RNA Sequencing-Mediated Transcriptome Analysis of Rice Plants in Endoplasmic Reticulum Stress Conditions. BMC Plant Biology, 14, 101. https://doi.org/10.1186/1471-2229-14-101

[37] Rasmussen, S., Barah, P., Suarez-Rodriguez, M.C., et al. (2013) Transcriptome Responses to Combinations of Stresses in Arabidopsis. Plant Physiology, 161, 1783-1794. https://doi.org/10.1104/pp.112.210773

[38] Raney, J., Reynolds, D., Elzinga, D., et al. (2014) Transcriptome Analysis of Drought Induced Stress in Chenopodium quinoa. American Journal of Plant Sciences, 5, 338-357. https://doi.org/10.4236/ajps.2014.53047

[39] Kudapa, H., Azam, S., Sharpe, A.G., et al. (2014) Comprehensive Transcriptome Assembly of Chickpea (Cicer arietinum L.) Using Sanger and Next Generation Sequencing Platforms: Development and Applications. PLoS ONE, 9, e86039. https://doi.org/10.1371/journal.pone.0086039

[40] Jain, D. and Chattopadhyay, D. (2010) Analysis of Gene Expression in Response to Water Deficit of Chickpea (Cicer arietinum L.) Varieties Differing in Drought Tolerance. BMC Plant Biology, 10, 24. https://doi.org/10.1186/1471-2229-10-24

[41] Xu, Y., Gao, S., Yang, Y., et al. (2013) Transcriptome Sequencing and Whole Genome Expression Profiling of Chrysanthemum under Dehydration Stress. BMC Genomics, 14, 662. https://doi.org/10.1186/1471-2164-14-662

[42] Zhu, Y.N., Shi, D.Q., Ruan, M.B., et al. (2013) Transcriptome Analysis Reveals Crosstalk of Responsive Genes to Multiple Abiotic Stresses in Cotton (Gossypium hirsutum L.). PLoS ONE, 8, e80218. https://doi.org/10.1371/journal.pone.0080218

[43] Trujillo, L.E., Sotolongo, M., Menendez, C., et al. (2008) SodERF3, a Novel Sugarcane Ethylene-Responsive Factor (ERF), Enhances Salt and Drought Tolerance When Overexpressed in Tobacco Plants. Plant and Cell Physiology, 49, 512-525. https://doi.org/10.1093/pcp/pcn025 
[44] Gygi, S.P., Rochon, Y., Franza, B.R., et al. (1999) Correlation between Protein and mRNA Abundance in Yeast. Molecular and Cellular Biology, 19, 1720-1730. https://doi.org/10.1128/MCB.19.3.1720

[45] Hakeem, K.R., Chandna, R., Ahmad, P., et al. (2012) Relevance of Proteomic Investigations in Plant Abiotic Stress Physiology. Omics, 16, 621-635.

https://doi.org/10.1089/omi.2012.0041

[46] Hossain, Z. and Komatsu, S. (2013) Contribution of Proteomic Studies towards Understanding Plant Heavy Metal Stress Response. Frontiers in Plant Science, 3, 310. https://doi.org/10.3389/fpls.2012.00310

[47] Anderson, L. and Seilhamer, J. (1997) A Comparison of Selected mRNA and Protein Abundances in Human Liver. Electrophoresis, 18, 533-537. https://doi.org/10.1002/elps.1150180333

[48] Komili, S. and Silver, P.A. (2008) Coupling and Coordination in Gene Expression Processes: A Systems Biology View. Nature Reviews Genetics, 9, 38-48. https://doi.org/10.1038/nrg2223

[49] Halbeisen, R.E. and Gerber, A.P. (2012) Correction: Stress-Dependent Coordination of Transcriptome and Translatome in Yeast. PLOS Biology, 10, 10. https://doi.org/10.1371/annotation/7462bca2-5358-43c8-be2e-94e8a8f46159

[50] Juntawong, P., Girke, T., Bazin, J., et al. (2014) Translational Dynamics Revealed by Genome-Wide Profiling of Ribosome Footprints in Arabidopsis. Proceedings of the National Academy of Sciences of the United States of America, 111, E203-E212. https://doi.org/10.1073/pnas.1317811111

[51] Yanguez, E., Castro-Sanz, A.B., Fernandez-Bautista, N., et al. (2013) Analysis of Genome-Wide Changes in the Translatome of Arabidopsis Seedlings Subjected to Heat Stress. PLoS ONE, 8, e71425. https://doi.org/10.1371/journal.pone.0071425

[52] Alvarez, S., Berla, B.M., Sheffield, J., et al. (2009) Comprehensive Analysis of the Brassica juncea Root Proteome in Response to Cadmium Exposure by Complementary Proteomic Approaches. Proteomics, 9, 2419-2431. https://doi.org/10.1002/pmic.200800478

[53] Hossain, Z., Hajika, M. and Komatsu, S. (2012) Comparative Proteome Analysis of High and Low Cadmium Accumulating Soybeans under Cadmium Stress. Amino Acids, 43, 2393-2416. https://doi.org/10.1007/s00726-012-1319-6

[54] Hossain, Z., Makino, T. and Komatsu, S. (2012) Proteomic Study of $\beta$-Aminobutyric Acid-Mediated Cadmium Stress Alleviation in Soybean. Journal of Proteomics, 75, 4151-4164. https://doi.org/10.1016/j.jprot.2012.05.037

[55] Hradilova, J., Rehulka, P., Rehulkova, H., et al. (2010) Comparative Analysis of Proteomic Changes in Contrasting Flax Cultivars upon Cadmium Exposure. Electrophoresis, 31, 421-431. https://doi.org/10.1002/elps.200900477

[56] Semane, B., Dupae, J., Cuypers, A., et al. (2010) Leaf Proteome Responses of Arabidopsis thaliana Exposed to Mild Cadmium Stress. Journal of Plant Physiology, 167, 247-254. https://doi.org/10.1016/j.jplph.2009.09.015

[57] Ahsan, N., Nakamura, T. and Komatsu, S. (2012) Differential Responses of Microsomal Proteins and Metabolites in Two Contrasting Cadmium (Cd) Accumulating Soybean Cultivars under Cd Stress. Amino Acids, 42, 317-327. https://doi.org/10.1007/s00726-010-0809-7

[58] Alves, M., Moes, S., Jeno, P., et al. (2011) The Analysis of Lupinus albus Root Proteome Revealed Cytoskeleton Altered Features Due to Long-Term Boron Deficiency. Journal of Proteomics, 74, 1351-1363.

https://doi.org/10.1016/j.jprot.2011.03.002 
[59] Wang, R., Gao, F., Guo, B.Q., et al. (2013) Short-Term Chromium-Stress-Induced Alterations in the Maise Leaf Proteome. International Journal of Molecular Sciences, 14, 11125-11144. https://doi.org/10.3390/ijms140611125

[60] Sharmin, S.A., Alam, I., Kim, K.H., et al. (2012) Chromium-Induced Physiological and Proteomic Alterations in Roots of Miscanthus sinensis. Plant Science, 187, 113-126. https://doi.org/10.1016/j.plantsci.2012.02.002

[61] Weckwerth, W. and Kahl, G. (2013) The Handbook of Plant Metabolomics. Wiley-Blackwell, Hoboken. https://doi.org/10.1002/9783527669882

[62] Urano, K., Maruyama, K., Ogata, Y., et al. (2009) Characterisation of the ABA-Regulated Global Responses to Dehydration in Arabidopsis by Metabolomics. The Plant Journal, 57, 1065-1078. https://doi.org/10.1111/j.1365-313X.2008.03748.x

[63] Skirycz, A., De Bodt, S., Obata, T., et al. (2010) Developmental Stage Specificity and the Role of Mitochondrial Metabolism in the Response of Arabidopsis Leaves to Prolonged Mild Osmotic Stress. Plant Physiology, 152, 226-244. https://doi.org/10.1104/pp.109.148965

[64] Witt, S., Galicia, L., Lisec, J., et al. (2012) Metabolic and Phenotypic Responses of the Greenhouse-Grown Maise Hybrids to Experimentally Controlled Drought Stress. Molecular Plant, 5, 401-417. https://doi.org/10.1093/mp/ssr102

[65] Bowne, J.B., Erwin, T.A., Juttner, J., et al. (2012) Drought Responses of Leaf Tissues from Wheat Cultivars of Differing Drought Tolerance at the Metabolite Level. $\mathrm{Mo}$ lecular Plant, 5, 418-429. https://doi.org/10.1093/mp/ssr114

[66] Verslues, P.E. and Juenger, T.E. (2011) Drought, Metabolites, and Arabidopsis Natural Variation: A Promising Combination for Understanding Adaptation to Water-Limited Environments. Current Opinion in Plant Biology, 14, 240-245. https://doi.org/10.1016/j.pbi.2011.04.006

[67] Caldana, C., Degenkolbe, T., Inostroza, A.C., et al. (2011) High-Density Kinetic Analysis of the Metabolomic and Transcriptomic Response of Arabidopsis to Eight Environmental Conditions. The Plant Journal, 67, 869-884. https://doi.org/10.1111/j.1365-313X.2011.04640.x

[68] Kusano, M., Tohge, T., Fukushima, A., et al. (2011) Metabolomics Reveals Comprehensive Reprogramming Involving Two Independent Metabolic Responses of Arabidopsis to UV-B Light. The Plant Journal, 67, 354-369.

https://doi.org/10.1111/j.1365-313X.2011.04599.x

[69] Tohge, T., Kusano, M., Fukushima, A., et al. (2011) Transcriptional and Metabolic Programs Following Exposure of Plants to UV-B Irradiation. Plant Signaling \& Behavior, 6, 1987-1992. https://doi.org/10.4161/psb.6.12.18240

[70] Araujo, W.L., Ishizaki, K., Nesi, A.N., et al. (2011) Analysis of a Range of Catabolic Mutants Provide Evidence that Phytanoyl-Coenzyme A Does Not Act as a Substrate of the Electron-Transfer Flavoprotein/Electron-Transfer Flavoprotein: Ubiquinone-Oxidoreductase Complex in Arabidopsis during Dark-Induced Senescence. Plant Physiology, 157, 55-69. https://doi.org/10.1104/pp.111.182188

[71] Joshi, V., Joung, J.G., Fei, Z., et al. (2010) Interdependence of Threonine, Methionine and Isoleucine Metabolism in Plants: Accumulation and Transcriptional Regulation under Abiotic Stress. Amino Acids, 39, 933-947. https://doi.org/10.1007/s00726-010-0505-7

[72] Araujo, W.L., Tohge, T., Ishizaki, K., et al. (2011) Protein Degradation: An Alternative Respiratory Substrate for Stressed Plants. Trends in Plant Science, 16, 489-498. https://doi.org/10.1016/j.tplants.2011.05.008

[73] Wochniak, E.U., Luedemann, A., Kopka, J., et al. (2003) Parallel Analysis of Tran- 
script and Metabolic Profiles: A New Approach in Systems Biology. EMBO Reports, 4, 989-993. https://doi.org/10.1038/sj.embor.embor944

[74] Zeng, J., Liu, Y., Liu, W., et al. (2013) Integration of Transcriptome, Proteome and Metabolism Data Reveals the Alkaloids Biosynthesis in Macleayacordata and Macleayamicrocarpa. PLoS ONE, 8, e53409. https://doi.org/10.1371/journal.pone.0053409

[75] Lan, P., Li, W. and Schmidt, W. (2012) Complementary Proteome and Transcriptome Profiling in Phosphate-Deficient Arabidopsis Roots Reveals Multiple Levels of Gene Regulation. Molecular \& Cellular Proteomics, 11, 1156-1166. https://doi.org/10.1074/mcp.M112.020461

[76] Colmsee, C., Mascher, M., Czauderna, T., et al. (2012) OPTIMAS-DW: A Comprehensive Transcriptomics, Metabolomics, Ionomics, Proteomics and Phenomics Data Resource for Maise. BMC Plant Biology, 12, 245. https://doi.org/10.1186/1471-2229-12-245

[77] Amiour, N., Imbaud, S., Clement, G., et al. (2012) The Use of Metabolomics Integrated with Transcriptomic and Proteomic Studies for Identifying Key Steps Involved in the Control of Nitrogen Metabolism in Crops Such as Maise. Journal of Experimental Botany, 63, 5017-5033. https://doi.org/10.1093/jxb/ers186

[78] Srivastava, V., Obudulu, O., Bygdell, J., et al. (2013) OnPLS Integration of Transcriptomic, Proteomic and Metabolomic Data Shows Multi-Level Oxidative Stress Responses in the Cambium of Transgenic hipI-Superoxide Dismutase Populus Plants. BMC Genomics, 14, 893. https://doi.org/10.1186/1471-2164-14-893

[79] Usadel, B., Obayashi, T., Mutwil, M., Giorgi, F.M., Bassel, G.W., Tanimoto, M., Chow, A., Steinhauser, D., Persson, S. and Provart, N.J. (2009) Co-Expression Tools for Plant Biology: Opportunities for Hypothesis Generation and Caveats. Plant, Cell \& Environment, 32, 1633-1651. https://doi.org/10.1111/j.1365-3040.2009.02040.x

[80] Hirai, M.Y., Yano, M., Goodenowe, D.B., Kanaya, S., Kimura, T., Awazuhara, M., Arita, M., Fujiwara, T. and Saito, K. (2004) Integration of Transcriptomics and Metabolomics for Understanding of Global Responses to Nutritional Stresses in Arabidopsis thaliana. Proceedings of the National Academy of Sciences of the United States of America, USA, 101, 10205-10210. https://doi.org/10.1073/pnas.0403218101

[81] Hirai, M.Y., Sugiyama, K., Sawada, Y., Tohge, T., Obayashi, T., Suzuki, A., Araki, R., Sakurai, N., Suzuki, H., Aoki, K., Goda, H., Nishizawa, O.I., Shibata, D. and Saito, K. (2007) Omics-Based Identification of Arabidopsis Myb Transcription Factors Regulating Aliphatic Glucosinolate Biosynthesis. Proceedings of the National Academy of Sciences of the United States of America, USA, 104, 6478-6483. https://doi.org/10.1073/pnas.0611629104

[82] Mao, L., Van, H.J.L., Dash, S. and Dickerson, J.A. (2009) Arabidopsis Gene Co-Expression Network and Its Functional Modules. BMC Bioinformatics, 10, 346. https://doi.org/10.1186/1471-2105-10-346

[83] Carrera, J., Rodrigo, G., Jaramillo, A. and Elena, S.F. (2009) Reverse-Engineering the Arabidopsis thaliana Transcriptional Network under Changing Environmental Conditions. Genome Biology, 10, R96. https://doi.org/10.1186/gb-2009-10-9-r96

[84] Lorenz, W.W., Alba, R., Yu, Y.S., Bordeaux, J.M., Simoes, M. and Dean, J.F. (2011) Microarray Analysis and Scale-Free Gene Networks Identify Candidate Regulators in Drought-Stressed Roots of Loblolly Pine (P. taeda L.). BMC Genomics, 12, 264. https://doi.org/10.1186/1471-2164-12-264

[85] Weston, D.J., Gunter, L.E., Rogers, A. and Wullschleger, S.D. (2008) Connecting 
Genes, Coexpression Modules, and Molecular Signatures to Environmental Stress Phenotypes in Plants. BMC Systems Biology, 2, 16.

https://doi.org/10.1186/1752-0509-2-16

[86] Weston, D.J., Karve, A.A., Gunter, L.E., Jawdy, S.S., Yang, X., Allen, S.M. and Wullschleger, S.D. (2011) Comparative Physiology and Transcriptional Networks Underlying the Heat Shock Response in Populus trichocarpa, Arabidopsis thaliana and Glycine max. Plant, Cell \& Environment, 34, 1488-1506.

https://doi.org/10.1111/j.1365-3040.2011.02347.x

[87] Kilian, J., Whitehead, D., Horak, J., Wanke, D., Weinl, S., Batistic, O., D’Angelo, C., Bornberg-Bauer, E., Kudla, J. and Harter, K. (2007) The AtGenExpress Global Stress Expression Data Set: Protocols, Evaluation and Model Data Analysis of UV-B Light, Drought and Cold Stress Responses. The Plant Journal, 50, 347-363. https://doi.org/10.1111/j.1365-313X.2007.03052.x

[88] Sun, X., Zou, Y., Nikiforova, V., Kurths, J. and Walther, D. (2010) The Complexity of Gene Expression Dynamics Revealed by Permutation Entropy. BMC Bioinformatics, 11, 607. https://doi.org/10.1186/1471-2105-11-607

[89] Vanderauwera, S., Zimmermann, P., Rombauts, S., Vandenabeele, S., Langebartels, C., Gruissem, W., Inze, D. and Van, B.F. (2005) Genome-Wide Analysis of Hydrogen Peroxide-Regulated Gene Expression in Arabidopsis Reveals a High Light-Induced Transcriptional Cluster Involved in Anthocyanin Biosynthesis. Plant Physiology, 139, 806-821. https://doi.org/10.1104/pp.105.065896

[90] Tognetti, V.B., Van, A.O., Morreel, K., Vandenbroucke, K., van, d.C.B., De, C.I., Chiwocha, S., Fenske, R., Prinsen, E., Boerjan, W., Genty, B., Stubbs, K.A., Inze, D. and Van, B.F. (2010) Perturbation of Indole-3-Butyric Acid Homeostasis by the UDP-glucosyltransferase UGT74E2 Modulates Arabidopsis Architecture and Water Stress Tolerance. Plant Cell, 22, 2660-2679. https://doi.org/10.1105/tpc.109.071316

[91] Vanderauwera, S., De, B.M., Van, d.S.N., van, d.C.B., Metzlaff, M. and Van, B.F. (2007) Silencing of Poly(ADP-ribose) Polymerase in Plants Alters Abiotic Stress Signal Transduction. Proceedings of the National Academy of Sciences of the United States of America, USA, 104, 15150-15155.

https://doi.org/10.1073/pnas.0706668104

[92] De Block, M., Verduyn, C., De Brouwer, D. and Cornelissen, M. (2005) Poly(ADP-ribose) Polymerase in Plants Affects Energy Homeostasis, Cell Death and Stress Tolerance. The Plant Journal, 41, 95-106. https://doi.org/10.1111/j.1365-313X.2004.02277.x

[93] Bashandy, T., Taconnat, L., Renou, J.P., Meyer, Y. and Reichheld, J.P. (2009) Accumulation of Flavonoids in an Ntrantrb Mutant Leads to Tolerance to UV-C. Molecular Plant, 2, 249-258. https://doi.org/10.1093/mp/ssn065

[94] Wilkins, O., Brautigam, K. and Campbell, M.M. (2010) Time of Day Shapes Arabidopsis Drought Transcriptomes. The Plant Journal, 63, 715-727.

https://doi.org/10.1111/j.1365-313X.2010.04274.x

[95] Cook, D., Fowler, S., Fiehn, O. and Thomashow, M.F. (2004) A Prominent Role for the CBF Cold Response Pathway in Configuring the Low-Temperature Metabolome of Arabidopsis. Proceedings of the National Academy of Sciences of the United States of America, USA, 101, 15243-15248.

https://doi.org/10.1073/pnas.0406069101

[96] Blank, L.M., Kuepfer, L. and Sauer, U. (2005) Large-Scale 13Cflux Analysis Reveals Mechanistic Principles of Metabolic Network Robustness to Null Mutations in Yeast. Genome Biology, 6, R49-R54. https://doi.org/10.1186/gb-2005-6-6-r49 
[97] Fernie, A.R., Geigenberger, P. and Stitt, M. (2005) Flux an Important, But Neglected, Component of Functional Genomics. Current Opinion in Plant Biology, 8, 174-182. https://doi.org/10.1016/j.pbi.2005.01.008

[98] Krishnan, P., Kruger, N.J. and Ratcliffe, R.G. (2005) Metabolite Fingerprinting and Profiling in Plants Using NMR. Journal of Experimental Botany, 56, 255-265. https://doi.org/10.1093/jxb/eri010 